\title{
AVAnCES EN LA identificaCión de las FUENTES DE ARCILla EXPLOTADAS POR LOS ALFAREROS DE LA COSTA SURCENTRAL DEL Perú durante la PRIMERA Mitad DEl PRIMER Milenio A.C.: UN ESTUDIO A PARTIR DEL ANÁLISIS POR LA-ICP-MS DE VASIJAS de Cerámica de Puerto Nuevo y muestras de arcillas de los VAlles de Pisco E ICA
}

\author{
Jalh Dulanto \\ https://orcid.org/0000-0003-0528-1860 \\ Departamento de Humanidades, Pontificia Universidad Católica del Perú \\ jdulant@pucp.pe \\ Isabelle Druc \\ Departamento de Antropología, University of Wisconsin - Madison \\ icdruc@wisc.edu \\ Laure Dussubieux \\ Field Museum of Natural History \\ ldussubieux@fieldmuseum.org \\ Enrique Guadalupe \\ Departamento de Ciencias e Ingeniería, Pontificia Universidad Católica del Perú \\ eguadal@pucp.pe \\ Alejandro Rey de Castro \\ Departamento de Humanidades, Pontificia Universidad Católica del Perú \\ areydecas@gmail.com
}

\begin{abstract}
Resumen
En este articulo presentamos nuestros avances en el estudio comparativo de la composición quimica de una muestra representativa de fragmentos de vasijas de cerámica de Puerto Nuevo y de arcillas de los valles de Pisco e Ica. Con esto buscamos identificar las fuentes explotadas por los alfareros que elaboraron las vasijas de cerámica usadas y desechadas en este sitio. De esta manera buscamos contribuir a la comprensión de las complejas interacciones sociales en las que estuvieron involucradas las poblaciones de la costa sur durante la primera mitad del primer milenio antes de nuestra era. Mostramos de qué maneras un estudio minucioso de los rasgos tecnológicos, morfológicos y decorativos de las vasijas, junto con un examen detallado de sus pastas —en el que se combina el análisis de la composición mineral (con lupa digital de cortes frescos y con microscopio petrográfico de láminas delgadas) y el análisis de la composición química (por $L A-I C P-M S)$ - permiten acercarnos a estos objetivos.
\end{abstract}

Palabras clave: LA-ICP-MS, vasijas de cerámica, fuentes de arcilla, Horizonte Temprano, Formativo, Costa Sur, Perú. 


\begin{abstract}
ADVANCES IN THE IDENTIFICATION OF THE CLAY SOURCES EXPLOITED BY THE POTTERS FROM THE SOUTHCENTRAL COAST OF PERU DURING THE FIRST HALF OF THE FIRST MILLENNIUM BC: A STUDY USING LA-ICP-MS ANALYSIS OF CERAMIC VESSELS FROM PUERTO NUEVO AND CLAY SAMPLES FROM THE PISCO AND ICA VALLEYS
\end{abstract}

In this article we present our advances in the comparative study of the chemical composition of a representative sample of pottery vessels from Puerto Nuevo and clays from the Pisco and Ica valleys. This is done in order to identify the clay sources exploited by the potters who produced the ceramic vessels that were used and discarded at this site. We seek, by such means, to contribute to the understanding of the complex social interactions involving the populations of the southern coast during the first half of the first millennium before our era. We show how a thorough examination of the technological, morphological and decorative features of these vessels, together with a detailed characterization of their pastes, combining both mineralogical (digital microscopy and petrography) and chemical (LA-ICP-MS) compositional analyses, allow us to approach these objectives.

Keywords: LA-ICP-MS, pottery vessels, clay sources, Early Horizon, Formative, South Coast, Peru.

\title{
1. Introducción
}

Durante la primera mitad del primer milenio antes de nuestra era (específicamente entre los siglos $\mathrm{X}-\mathrm{V}$ a.C.), las poblaciones de la costa surcentral del Perú ${ }^{1}$ (Fig. 1) produjeron, distribuyeron y consumieron vasijas de cerámica que variaban estilísticamente de un valle a otro, e incluso dentro del mismo valle. En el valle de Pisco estas vasijas suelen ser asignadas a diferentes estilos y, por tanto, tienen distintos nombres otorgados por diversos investigadores.

Así, por ejemplo, a partir del material recuperado durante sus excavaciones en varios sitios de Paracas, Engel $(1966,1991)$ reconoce la existencia de dos «estilos» a los que denomina Disco Verde y Chavín, mientras que García y Pinilla (1995) — a partir de la comparación de las vasijas asignadas por Engel a estos estilos con vasijas de otros sitios— reconocen la existencia de tres «fases» a las que denominaron Disco Verde, Puerto Nuevo y Karwa.

Sin embargo, ni la variación en la forma de las vasijas en cuestión, ni la distribución de esta variación en el espacio y en el tiempo, han sido estudiadas con la precisión y exactitud requeridas para identificar y caracterizar grupos de manera adecuada. Independientemente de que los grupos propuestos sean denominados «estilos», "períodos» o «fases», estos siguen siendo en gran medida subjetivos.

El Proyecto de Investigaciones Arqueológicas Paracas — dirigido por Jalh Dulanto - incluye un programa de estudio exhaustivo de las colecciones de fragmentos de vasijas de cerámica recuperados durante las excavaciones en varios sitios arqueológicos de la zona de Paracas, ubicada inmediatamente al sur del valle de Pisco, y tiene como uno de sus principales objetivos, definir adecuadamente estos grupos (Dulanto 2013; Dulanto y Accinelli 2013; Druc et al. 2017; Dulanto et al. 2019).

En este artículo presentamos nuestros avances en el estudio comparativo de la composición química de una muestra representativa de estos fragmentos y de las arcillas de los valles de Pisco e Ica, para identificar las fuentes explotadas por los alfareros que fabricaron las vasijas de cerámica usadas y desechadas en Puerto Nuevo, y de esta manera contribuir a la comprensión de las complejas interacciones sociales en las que estuvieron involucradas.

Asimismo, mostramos de qué maneras un estudio minucioso de los rasgos tecnológicos, morfológicos y decorativos de las vasijas, junto con un examen detallado de sus pastas, en el que se combina el análisis de la composición mineral —con lupa digital de cortes frescos y con microscopio petrográfico de láminas delgadas - y el análisis de la composición química — por espectrometría 
de masas con fuente de plasma de acoplamiento inductivo y ablación láser como sistema de introducción de muestra o LA-ICP-MS, por sus siglas en inglés - permiten acercarnos a estos objetivos.

\section{Puerto Nuevo en el contexto de los siglos X al V a.C. en los Andes Centrales}

Puerto Nuevo es uno de los pocos sitios arqueológicos de la costa surcentral del Perú con ocupaciones de los siglos X al V a.C. que han sido excavados por arqueólogos (Engel 1966, 1991; García 2009; Dulanto 2013). Está ubicado en un área costera desértica, más bien marginal, entre los valles de los ríos Pisco e Ica, pero mucho más cerca de las tierras agrícolas del valle bajo del río Pisco (15 kilómetros hacia el norte) que de las del valle bajo o el valle medio del río Ica (respectivamente 135 kilómetros y 60 kilómetros hacia el sur) (Fig. 1).

Puerto Nuevo tiene hoy en día la apariencia de una concentración de restos arqueológicos de aproximadamente 300 metros de diámetro, depositados sobre varios montículos naturales de menos de 1.5 metros de altura. Estos restos son producto de varias ocupaciones del lugar por comunidades de pescadores, quienes construyeron y habitaron una serie de casas circulares y cuadrangulares levantadas con material perecible, y subsistieron explotando los recursos del litoral y los humedales adyacentes (Fig. 2).

Engel (1963a, 1963b, 1963c, 1966, 1976, 1987, 1991) descubrió Puerto Nuevo y lo excavó por primera vez, identificando la presencia de vasijas de cerámica de dos «estilos» en los estratos de basura. Este investigador denominó a estos estilos Disco Verde y Chavín, y consideró que eran básicamente contemporáneos. Sin embargo, García y Pinilla (1995) determinaron posteriormente que las vasijas que Engel denominó Disco Verde y Chavín podían ser asignadas a tres «fases» de su cronología cerámica: el primero a la fase Disco Verde (1000-800 a.C.) y el segundo a las fases Puerto Nuevo (800-600 a.C.) y Karwa (600-400 a.C.).

No obstante, nuestras excavaciones en Puerto Nuevo y Disco Verde indican que es muy difícil poder ordenar en el tiempo los eventos de producción de las vasijas de estos diferentes estilos. Aunque el inicio de la tradición de producción de vasijas de estilo Disco Verde parece ser anterior al de vasijas de estilo Puerto Nuevo, y este a su vez parece ser anterior al de vasijas de estilo Karwa, el hecho es que en Puerto Nuevo encontramos fragmentos de estos tres estilos en los mismos estratos de basura, por lo que podemos suponer que estas piezas fueron elaboradas al mismo tiempo durante un periodo que aún no hemos logrado determinar (Dulanto 2013; Dulanto y Accinelli 2013).

Los estudios que estamos llevando a cabo con la colección de fragmentos de vasijas de cerámica de Puerto Nuevo están permitiendo caracterizar una realidad social mucho más compleja en el valle bajo de Pisco y la costa surcentral entre los siglos X y V a.C., marcada por la presencia de una mayor variación estilística que fue resultado de una intensificación de las interacciones a nivel local, regional e interregional. Estos estudios combinan el análisis estilístico con el análisis composicional para identificar grupos de vasijas que son muy probablemente locales (producidas en el valle bajo de Pisco), y diferenciarlos de casos atípicos que muy posiblemente son foráneas (confeccionadas en otros valles de la costa surcentral, e incluso otras subáreas de los Andes Centrales) (Druc et al. 2017; Dulanto et al. 2019).

Esta intensificación de interacciones a nivel local, regional e interregional concuerda con dos cambios generales que observamos en los Andes Centrales entre los siglos X y V a.C. y que están estrechamente relacionados uno con otro: por un lado, la aparición de élites regionales que lograron distinguirse del resto de la población y perpetuarse en el poder y, por el otro, la consolidación de una economía de prestigio caracterizada por la intensificación de la producción artesanal de bienes suntuarios y la expansión de las redes de intercambio a larga distancia de los mismos (Dulanto en prensa). 


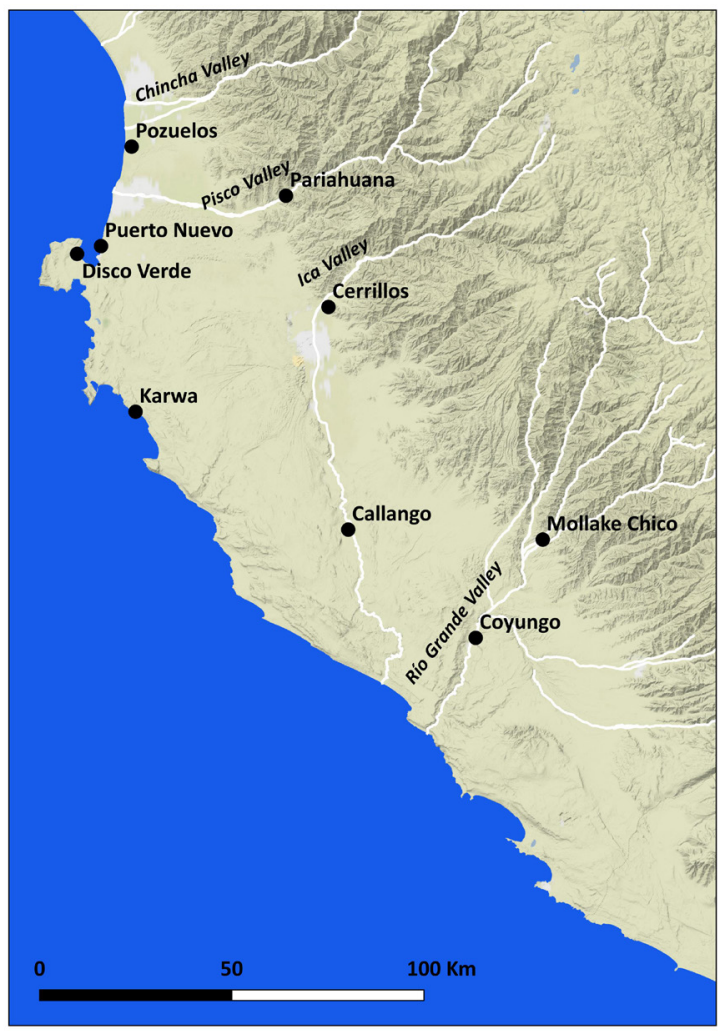

Figura 1. La costa surcentral del Perú con sus principales valles y sitios arqueológicos con ocupaciones de los siglos X-V a.C. (J. Dulanto).

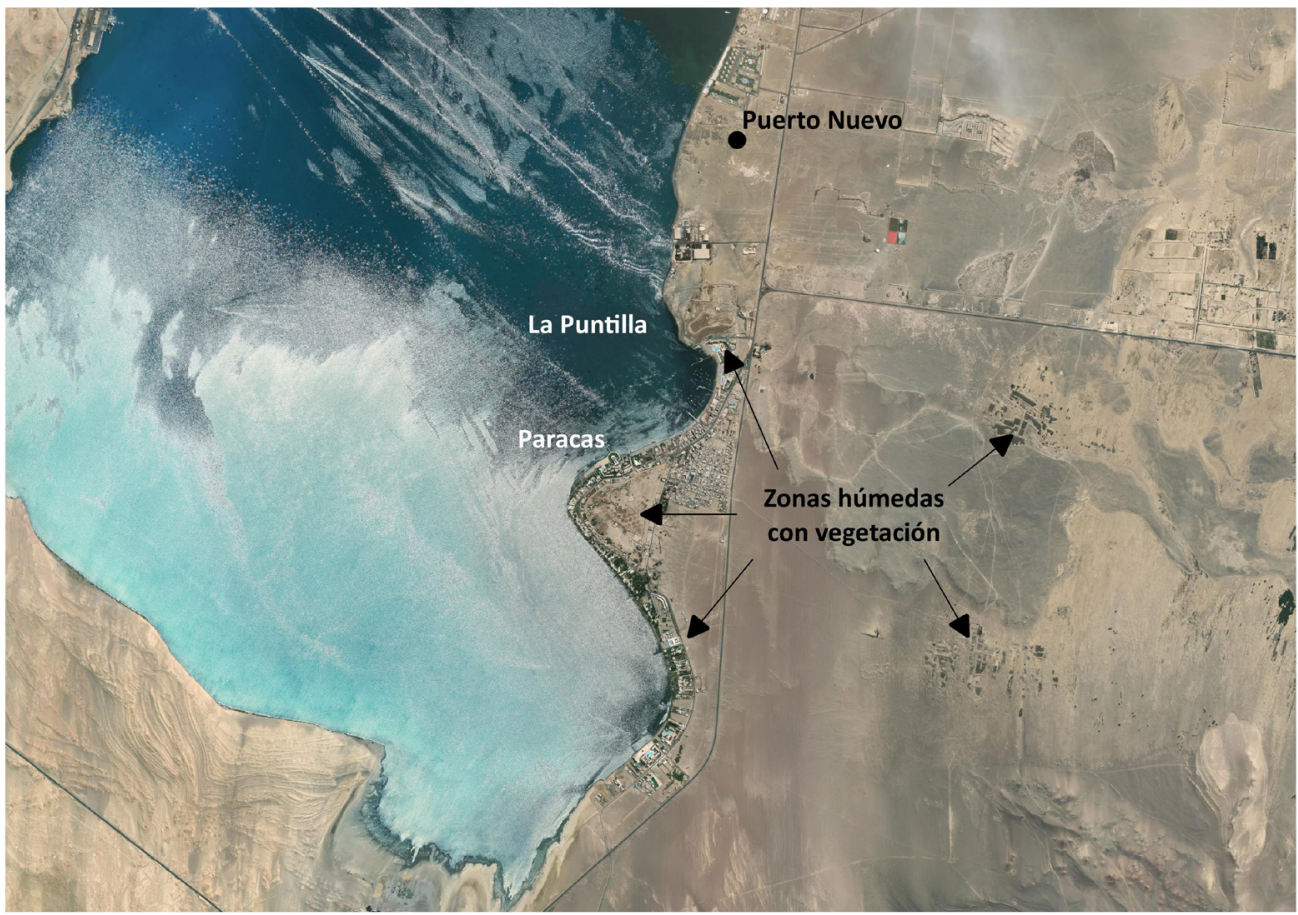

Figura 2. Ubicación del sitio arqueológico Puerto Nuevo en la zona de Paracas (J. Dulanto). 


\section{La muestra de vasijas de cerámica: Análisis estilístico y petrográfico}

La muestra analizada para este trabajo está compuesta por 403 fragmentos de vasijas de cerámica decoradas que fueron recuperadas durante nuestras excavaciones en Puerto Nuevo en $2013 .^{2}$

El análisis estilístico de estos fragmentos tomó en cuenta la correlación de variables tecnológicas, morfológicas y decorativas para definir grupos estilísticos e identificar especímenes estilísticamente atípicos. El análisis resultó en la definición de siete grupos estilísticos (GE I, II, III, IV, V, VI y VII), y la identificación de varios especímenes estilísticamente atípicos que no pueden ser asignados a ninguno de estos grupos.

Los grupos estilísticos I y II son variantes del estilo Disco Verde, tal y como fue definido por Engel $(1966,1991)$ y por García y Pinilla (1995). El grupo I corresponde a un conjunto de cuencos con y sin cuello, decorados con una o dos hileras de círculos estampados que van paralelas al borde de la vasija (Fig. 3). El grupo II comprende un conjunto de cuencos y botellas decorados con motivos geométricos — usualmente bandas, círculos, cruces, zigzags, etc.utilizando la técnica del negativo (Fig. 4). Los grupos estilísticos III, IV, V y VI son variantes del estilo Puerto Nuevo, tal y como es definido por estos mismos autores - aunque Engel lo denomina Chavín. El grupo III corresponde a un conjunto de cuencos decorados usualmente con cuatro almenas (Fig. 5). El grupo IV incluye un conjunto de cuencos y botellas decorados con motivos geométricos y figurativos utilizando la técnica de incisiones anchas y profundas, y pintura postcocción en zonas. Algunos de estos motivos evocan claramente tradiciones iconográficas norteńas (Cupisnique) y centrales (Ancón), mientras que otros evocan tradiciones locales más tardías (Paracas Medio y Paracas Tardío) (Fig. 6). El grupo V corresponde principalmente a un conjunto de cuencos decorados con motivos geométricos —usualmente bandas paralelas diagonales que se desprenden del labio- utilizando la técnica de pintura postcocción (Fig. 7). El grupo VI está integrado por un conjunto de botellas decoradas con bandas de dos o tres hileras de círculos impresos (Fig. 8). Finalmente, el grupo estilístico VII, que no está vinculado ni al estilo Disco Verde ni al estilo Puerto Nuevo, corresponde a un conjunto de cuencos y botellas elaboradas en estilos foráneos vinculados a la Costa Central, norcentral y norte, las cuales están decoradas con motivos geométricos y figurativos utilizando técnicas como la incisión, la impresión y el modelado, o una combinación de ellas (Fig. 9). Las botellas asa-estribo son comunes en este grupo. También lo son algunos cuencos prácticamente idénticos a los recuperados en Ancón en la Costa Central. Los especímenes estilísticamente atípicos incluyen una variedad de cuencos y botellas, locales y foráneos, que al parecer pertenecen a grupos estilísticos claramente minoritarios en la muestra analizada (ver Dulanto 2013; Druc et al. 2017). Estos especímenes muestran correlaciones interesantes con los grupos petrográficos que discutimos más adelante.

El análisis petrográfico de estos mismos fragmentos tomó en cuenta la correlación de variables de composición mineralógica y de tamaño y distribución de las inclusiones, así como de textura de la matriz arcillosa, para la definición de grupos petrográficos e identificación de especímenes petrográficamente atípicos. Todos los fragmentos fueron analizados primero con una lupa digital DinoLite Edge AM4815ZT con luz polarizada que permitió tomar fotografías de cortes frescos de la pasta a 30x y 130x. Las fotografías fueron procesadas para resaltar su contraste y analizadas utilizando el programa DinoCapture 2.0 Versión 1.5.0. Posteriormente, 120 de estos 403 fragmentos fueron cortados para obtener láminas delgadas que luego fueron analizadas en un microscopio con luz polarizada en «nicoles» paralelos y cruzados para estimar la proporción de los minerales y otras inclusiones presentes.

El análisis resultó en la definición de seis grupos petrográficos (GP A, B, C, D y F) y la identificación de varios especímenes petrográficamente atípicos que ya han sido descritos en detalle en otra publicación (Druc et al. 2017). Aquí nos vamos a limitar a reproducir las tablas que resumen las características de estos grupos petrográficos (Tablas 1 y 2). 

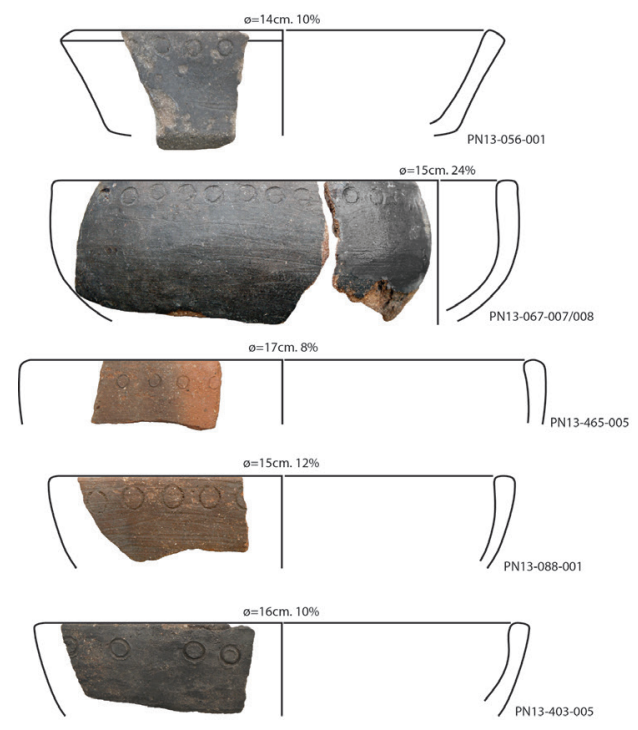
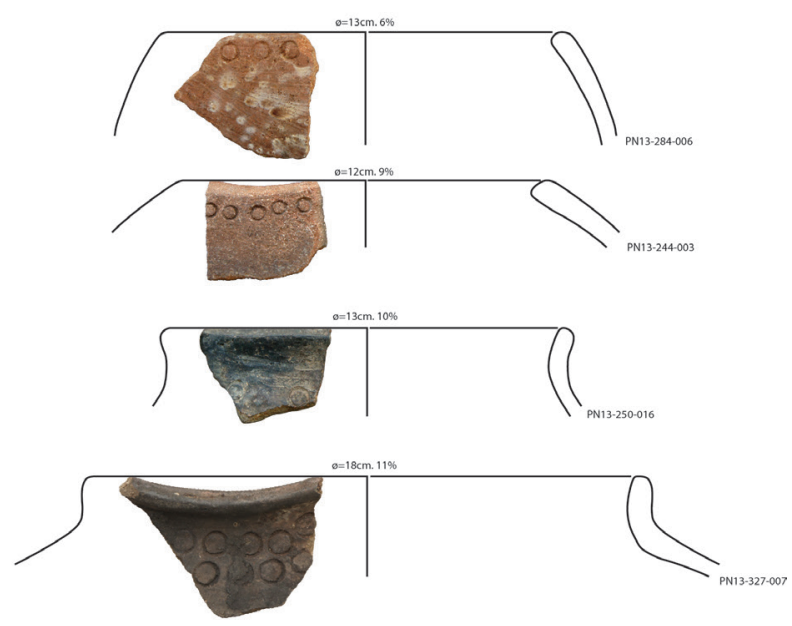

Figura 3. Vasijas del grupo estilístico I (J. Dulanto).
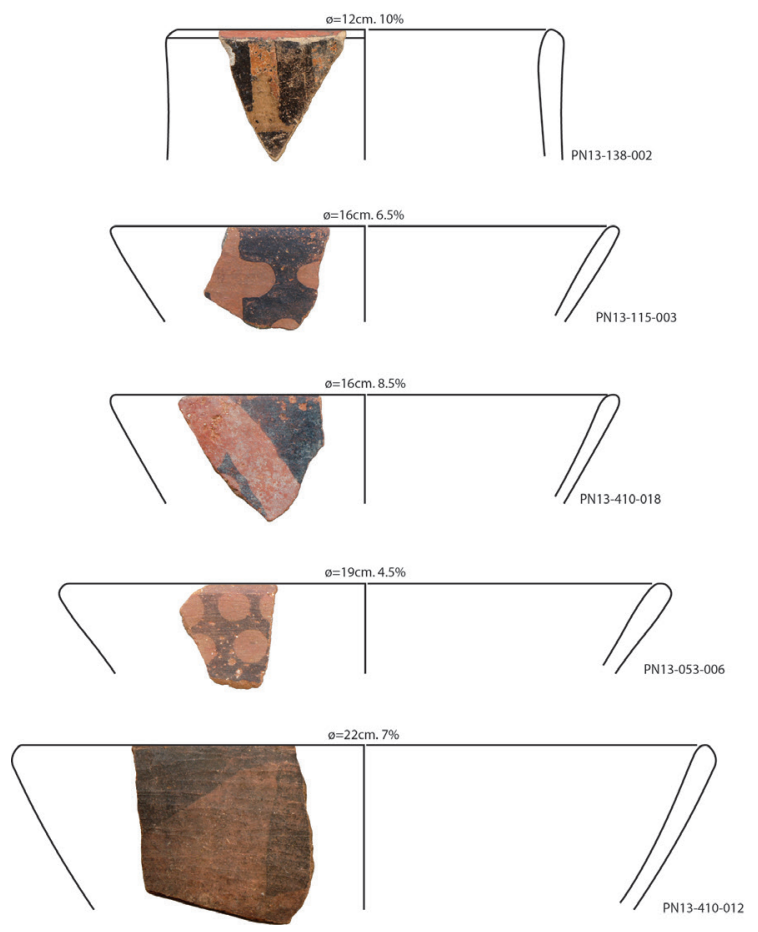

Figura 4. Vasijas del grupo estilistico II (J. Dulanto).
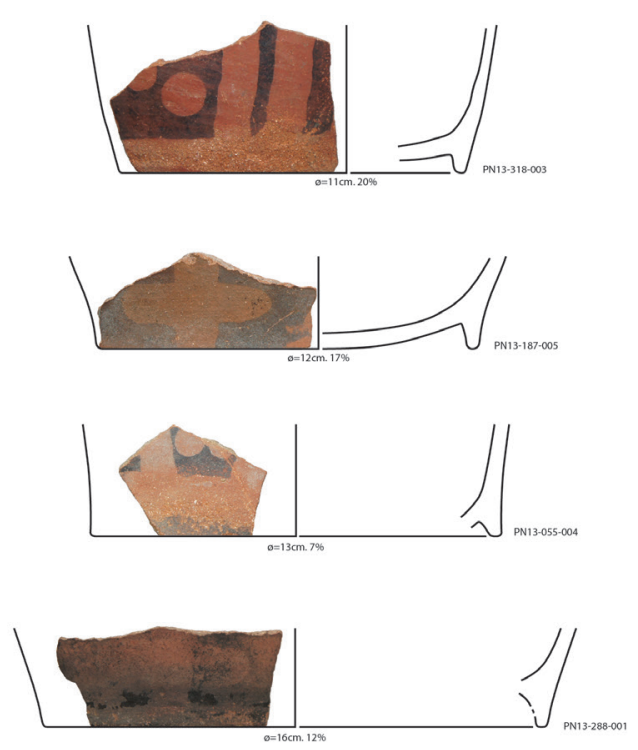

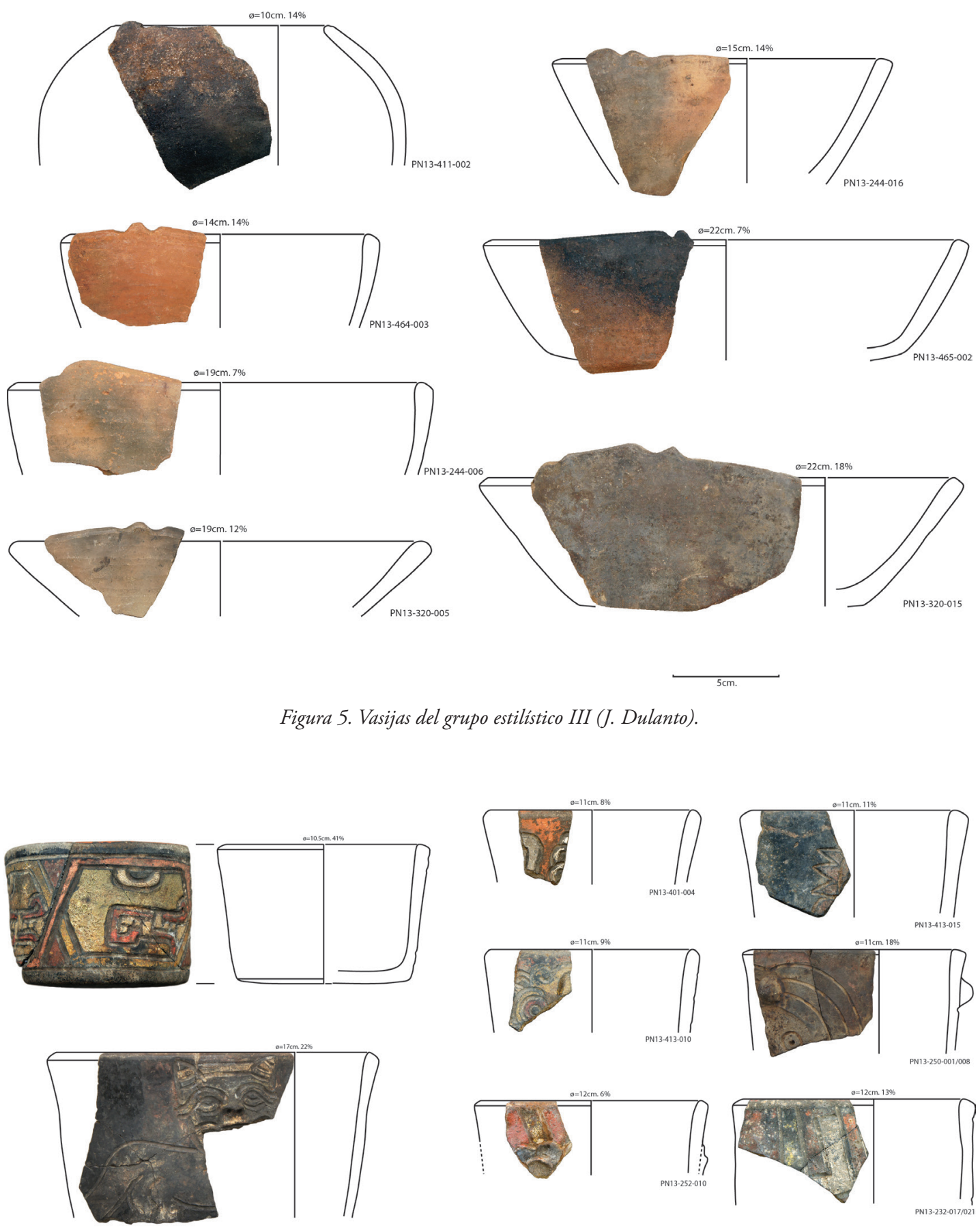

Figura 6. Vasijas del grupo estilístico IV (J. Dulanto). 

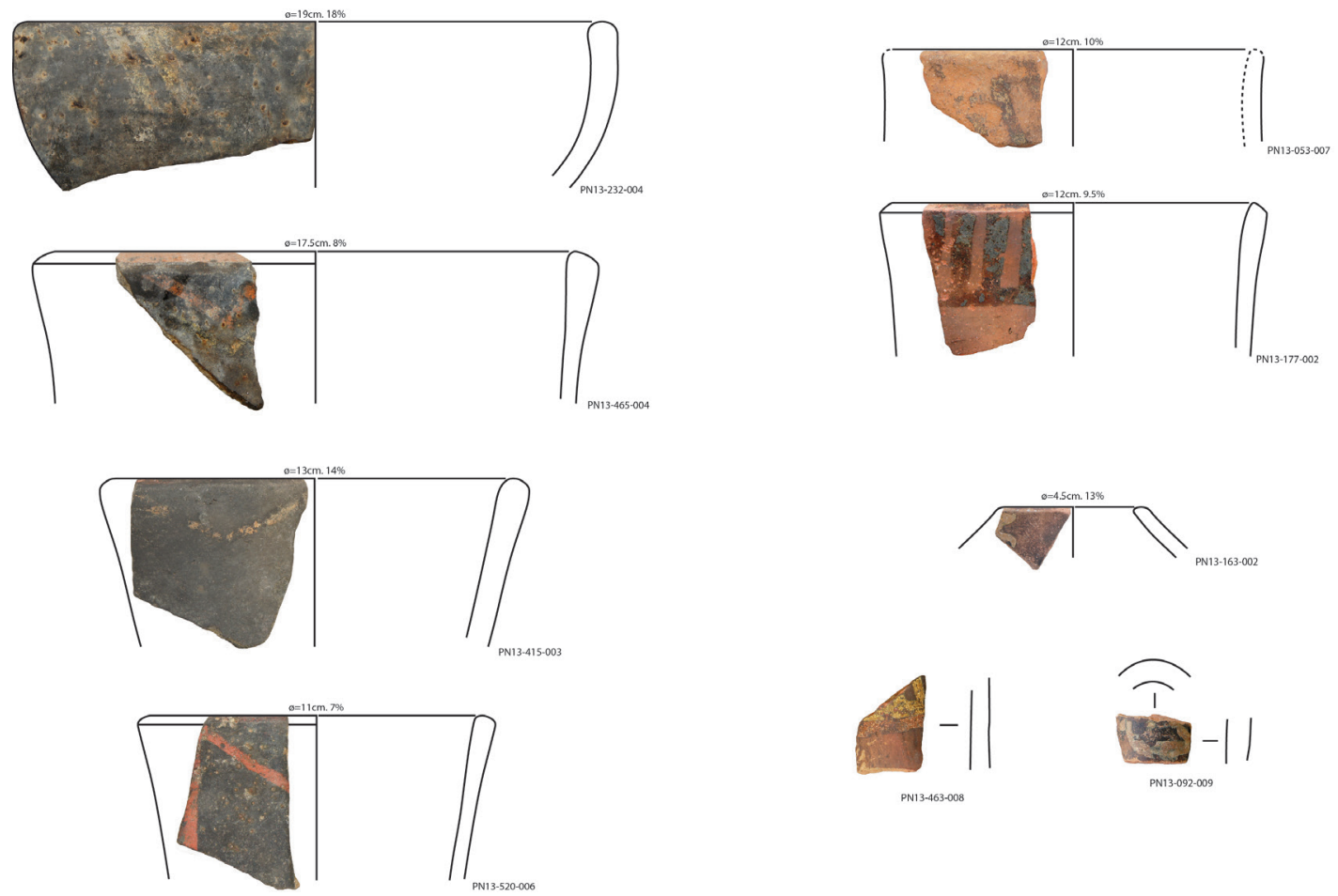

Figura 7. Vasijas del grupo estilístico V(J. Dulanto). 

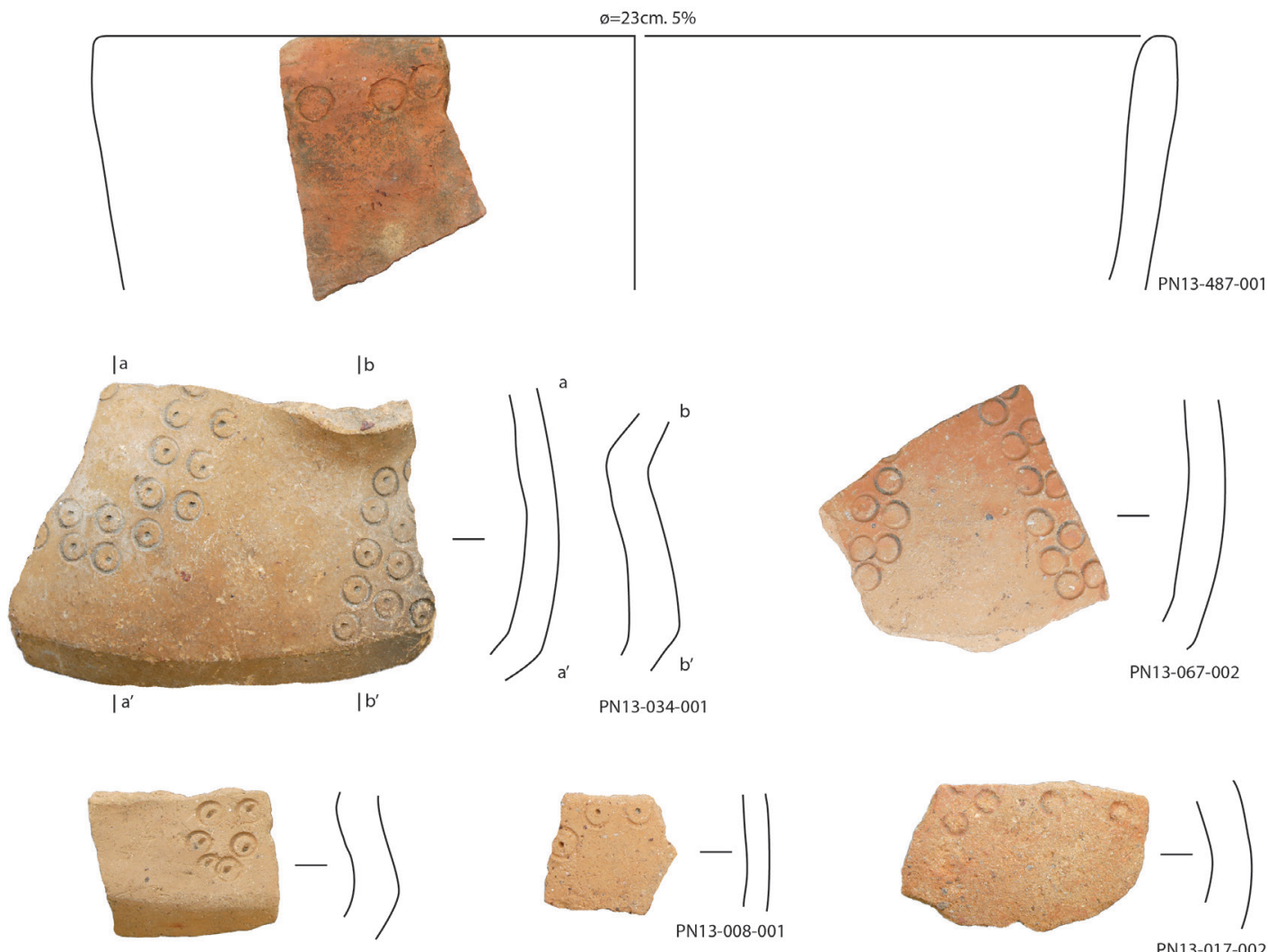

PN13-177-001

Figura 8. Vasijas del grupo estilistico VI (J. Dulanto).
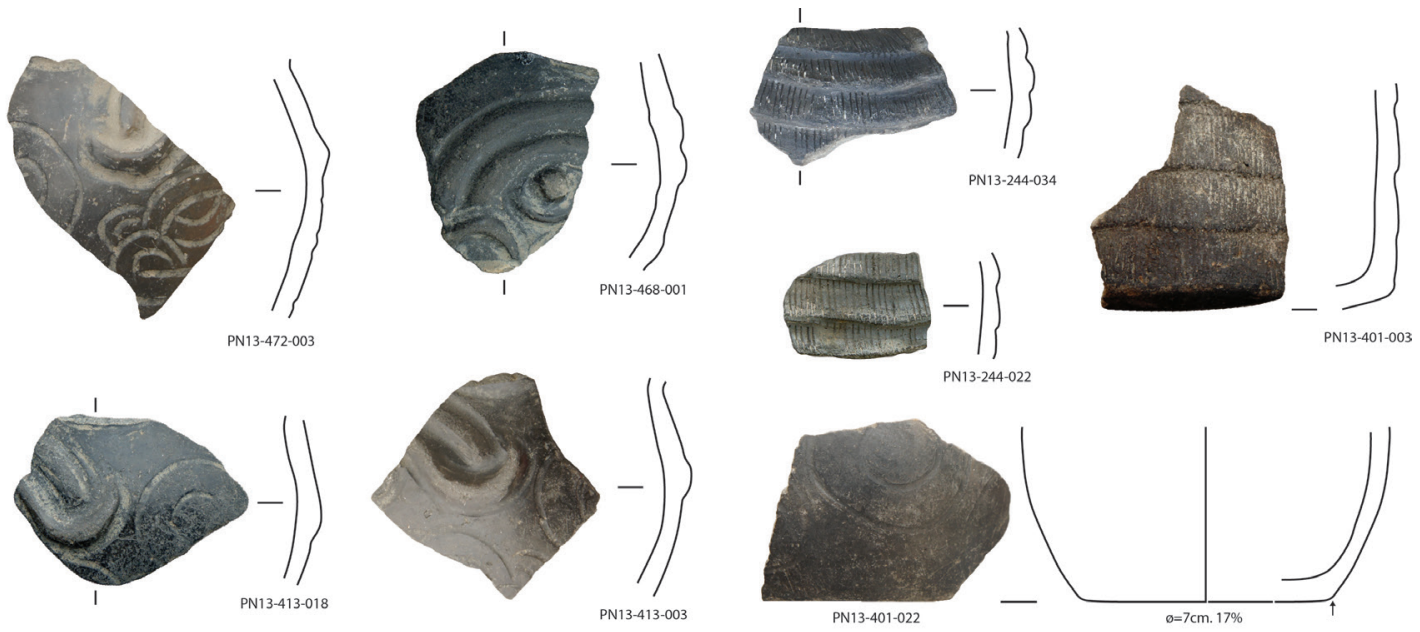

Figura 9. Vasijas del grupo estilístico VII (J. Dulanto). 


\begin{tabular}{|c|c|c|c|c|}
\hline $\begin{array}{c}\text { Grupo de } \\
\text { pasta }\end{array}$ & Granulométrica & $\%$ inclusiones & $\%$ inclusiones por tipo & $\begin{array}{c}\% \text { de } \\
\text { porosidad }\end{array}$ \\
\hline 1 & $\begin{array}{l}3 \% \text { de granos gruesos } \\
57 \% \text { de granos medianos } \\
24 \% \text { de granos finos } \\
16 \% \text { de granos muy finos }\end{array}$ & $20 \%$ & $\begin{array}{l}42 \% \text { de granos félsicos } \\
32 \% \text { de granos máficos } \\
26 \% \text { de litoclastos }\end{array}$ & $3 \%$ \\
\hline 2 & $\begin{array}{l}3 \% \text { de granos gruesos } \\
79 \% \text { de granos medianos } \\
12 \% \text { de granos finos } \\
6 \% \text { de granos muy finos }\end{array}$ & $30 \%$ & $\begin{array}{l}63 \% \text { de granos félsicos } \\
16 \% \text { de granos máficos } \\
20 \% \text { de litoclastos } \\
1 \% \text { de granos opacos }\end{array}$ & $3 \%$ \\
\hline 3 & $\begin{array}{l}3 \% \text { de granos gruesos } \\
60 \% \text { de granos medianos } \\
25 \% \text { de granos finos } \\
12 \% \text { de granos muy finos }\end{array}$ & $20 \%$ & $\begin{array}{l}\text { 44\% de granos félsicos } \\
18 \% \text { de granos máficos } \\
35 \% \text { de litoclastos } \\
3 \% \text { de óxidos }\end{array}$ & $3 \%-5 \%$ \\
\hline 4 & $\begin{array}{l}3 \% \text { de granos gruesos } \\
55 \% \text { de granos medianos } \\
21 \% \text { de granos finos } \\
21 \% \text { de granos muy finos }\end{array}$ & $15 \%$ & $\begin{array}{l}\text { 47\% de granos félsicos } \\
17 \% \text { de granos máficos } \\
28 \% \text { de litoclastos } \\
2 \% \text { de granos opacos } \\
5 \% \text { de óxidos } \\
1 \% \text { de epidotas }\end{array}$ & $3 \%$ \\
\hline 5 & $\begin{array}{l}2 \% \text { de granos gruesos } \\
71 \% \text { de granos medianos } \\
22 \% \text { de granos finos } \\
5 \% \text { de granos muy finos }\end{array}$ & $20 \%$ & $\begin{array}{l}47 \% \text { de granos félsicos } \\
23 \% \text { de granos máficos } \\
29 \% \text { de litoclastos } \\
1 \% \text { de granos opacos }\end{array}$ & $3 \%-5 \%$ \\
\hline 6 & $\begin{array}{l}1 \% \text { de granos gruesos } \\
57 \% \text { de granos medianos } \\
24 \% \text { de granos finos } \\
18 \% \text { de granos muy finos }\end{array}$ & $15 \%$ & $\begin{array}{l}41 \% \text { de granos félsicos } \\
29 \% \text { de granos máficos } \\
28 \% \text { de litoclastos } \\
2 \% \text { de granos opacos }\end{array}$ & $3 \%$ \\
\hline
\end{tabular}

Tabla 1. Principales características de los seis grupos petrográficos identificados para las vasijas decoradas de Puerto Nuevo a partir del análisis microscópico con lupa digital (A. Rey de Castro). 


\begin{tabular}{|c|c|c|c|c|c|c|c|c|}
\hline $\begin{array}{c}\text { Grupo } \\
\text { petrográfico }\end{array}$ & $\mathrm{n}$ & Antiplástico & Litoclastos & Minerales & $\begin{array}{l}\text { Bio- } \\
\text { clastos }\end{array}$ & $\begin{array}{l}\text { Mat. } \\
\text { org. }\end{array}$ & $\begin{array}{l}\text { carb/ } \\
\text { bio- } \\
\text { carb }\end{array}$ & Matriz \\
\hline 1 & 15 & $\begin{array}{l}\text { sedimento } \\
\text { granítico/tona- } \\
\text { lítico } \\
\text { rico en bt y } \\
\text { anf. }\end{array}$ & $\begin{array}{l}\text { g, sba } \\
\text { granito-grano- } \\
\text { diorita, tonalita, } \\
\text { metagranito, } \\
\text { mica esquisto, }\end{array}$ & $\begin{array}{l}\text { qz, pl, fd, bt, } \\
\text { hn, px, op, cl } \\
\text { f-m }\end{array}$ & - & - & - & $\begin{array}{l}\text { lpb } \\
\text { micácio, } \\
\text { ferr }\end{array}$ \\
\hline 2 & 11 & $\begin{array}{l}\text { sedimento } \\
\text { granítico }\end{array}$ & $\begin{array}{l}\text { g, alt sba-sbr } \\
\text { granito } \\
\text { (chert) }\end{array}$ & $\begin{array}{l}\text { m-g, alt } \\
\text { qz, fd, (pl), bt, } \\
\text { (hn), ep, cl, } \\
\text { msc, op }\end{array}$ & (x) & (x) & - & cf-lpb \\
\hline 3 & 28 & arena mixta & $\begin{array}{l}\text { andesita, chert, } \\
\text { arenisca, cuarzita, } \\
\text { metagranito } \\
\text { f-g alt }\end{array}$ & $\begin{array}{l}\text { f-g alt } \\
\text { qz, fd, pl, micas, } \\
\text { ox }\end{array}$ & $\mathrm{x}$ & $\mathrm{x}$ & $\mathrm{x}$ & $\begin{array}{l}\text { granular } \\
\text { lpb-cf }\end{array}$ \\
\hline 4 & 18 & $\begin{array}{l}\text { arena } \\
\text { sedimento- } \\
\text { granítica }\end{array}$ & $\begin{array}{l}\text { g } \\
\text { (arenisca, chert, } \\
\text { granito) }\end{array}$ & $\begin{array}{l}\text { f, alt } \\
\text { qz, fd, pl, bt, } \\
\text { hn, ox, op, ep }\end{array}$ & $\mathrm{x}$ & - & $\mathrm{x}$ & $\begin{array}{l}\text { pocas } \\
\text { incl. }\end{array}$ \\
\hline 5 & 23 & $\begin{array}{l}\text { arena volcáno- } \\
\text { sedimentaria }\end{array}$ & $\begin{array}{l}\text { f-g alt. } \\
\text { cuarcita, arenisca, } \\
\text { andesita }\end{array}$ & qz, pl, fd, hn, bt & - & $\begin{array}{l}x x \\
\text { caro- } \\
\text { fita }\end{array}$ & (x) & lpb-cf \\
\hline 6 & 12 & $\begin{array}{l}\text { arena } \\
\text { cuarcítica }\end{array}$ & $\begin{array}{l}\text { f-g } \\
\text { cuarcita } \\
\text { (granito) }\end{array}$ & $\begin{array}{l}\text { f-m } \\
q z, f d, p l, b t, \\
h n, p x, o x\end{array}$ & - & $\mathrm{x}$ & $\mathrm{x}$ & $\begin{array}{l}\text { ferr-op } \\
\text { limoso }\end{array}$ \\
\hline P atípicos & 13 & $\begin{array}{l}\text { sedimentos } \\
\text { arenosos } \\
\text { mixtos }\end{array}$ & Varios & Varios & Varios & Varios & Varios & Varios \\
\hline
\end{tabular}

Tabla 2. Principales caracteristicas de los seis grupos petrográficos identificados para las vasijas decoradas de Puerto Nuevo a partir del análisis petrográfico de láminas delgadas (GP = grupo petrográfico; atip = espécimen con composición atipica; ang = angular; $s b a=$ subangular; $s b r=$ subredondo; $q z=$ cuarzo; $p l=$ plagioclasa $; f d=$ feldespato; $b t$ $=$ biotita $;$ hn = hornblenda $; p x=$ piroxeno; op = opaco; $c l=$ clorita $; e p=$ epidota $;$ msc = moscovita $;$ ox =oxidos; $f=$ fino; $m=$ mediano; $g=$ grueso; mat org = material orgánico; $c a r b=$ carbonatos $/$ calcitalbiocarbonatos; $l p b=p$ seudolepidoblastico; $f f=$ criptofilitosa ferr $=$ ferruginoso; alt $=$ alterado $(I$. Druc $)$. 


\section{Los valles de Pisco e Ica y las muestras de arcilla}

Dos de los autores de este artículo - Enrique Guadalupe y Jalh Dulanto- recorrieron el valle bajo del río Pisco y el valle medio del río Ica en busca de fuentes de arcilla que pudieran haber sido explotadas por los alfareros que confeccionaron las vasijas de cerámica utilizadas y desechadas en Puerto Nuevo. En total recolectamos treinta muestras procedentes de diferentes tipos de yacimientos que contenían depósitos arcillosos que podrían haber sido explotados durante el primer milenio antes de nuestra era. Es importante notar que ninguno de ellos era una cantera de arcilla con evidencias arqueológicas de haber sido explotada en tiempos prehispánicos, pero esto no es en sí mismo un impedimento para distinguir entre arcillas de diferentes altitudes del fondo del valle y quebradas laterales que puedan tener firmas químicas diferentes. Como se puede ver en la siguiente figura, las muestras del valle de Pisco provienen de la sección más baja del valle bajo, mientras que todas las del valle de Ica provienen de la sección más alta del valle bajo y de la sección más baja del valle medio. En ambos casos tomamos como límite entre el valle bajo y el valle medio el punto donde el valle se estrecha, el cual suele coincidir con la zona en la que se ubican las bocatomas de los canales de irrigación de periodos más tardíos.

\section{Correlación de grupos estilísticos y grupos petrográficos y comparación de grupos petrográ- ficos con muestras de arcilla}

Los grupos estilísticos y petrográficos muestran ciertas correlaciones que resultan ser particularmente interesantes. Como se puede apreciar en la Tabla 3, las vasijas de los grupos petrográficos A y B tienden a pertenecer a los grupos estilísticos I y II vinculados al estilo local Disco Verde. Por su parte, las vasijas de los grupos petrográficos $\mathrm{D}$ y F, tienden a ser de los grupos estilísticos III, V y VI relacionados al estilo local Puerto Nuevo. Finalmente, las vasijas de los grupos petrográficos $\mathrm{C}$ y E, suelen ser parte del grupo estilístico IV — vinculado al estilo local Puerto Nuevo que reúne vasijas producidas localmente con técnicas del lugar pero que incorporan y adaptan motivos iconográficos de la Costa Central, norcentral y Norte-, como del grupo estilístico VII — vinculado a estilos foráneos de esas mismas regiones que reúne vasijas manufacturadas localmente, pero con técnicas foráneas. Cierta sobreposición entre estos tres conjuntos es resultado de la presencia de algunas vasijas del grupo petrográfico $\mathrm{D}$, que pertenecen a los grupos estilísticos IV y VII, y de algunas vasijas de los grupos petrográficos $\mathrm{C}$ y $\mathrm{E}$, que son parte del grupo estilístico V. Hay que destacar que casi todas las vasijas petrográficamente atípicas que hemos identificado son del grupo estilístico VII, vinculado a estilos foráneos de las regiones mencionadas. Estas últimas, podrían ser piezas producidas en esas regiones e importadas hasta la costa surcentral, o confeccionadas localmente por alfareros de esas otras regiones que vinieron hasta la costa surcentral y siguieron utilizando sus propias recetas para la preparación de la arcilla. Finalmente es importante notar que el resto de vasijas estilísticamente atípicas que hemos identificado pertenecen a los grupos petrográficos $\mathrm{A}, \mathrm{B}$ y F, y podrían ser ejemplos de estilos locales de la costa surcentral poco conocidos y poco representados en la muestra de Puerto Nuevo. 


\begin{tabular}{|c|c|c|c|c|c|c|c|c|}
\hline \multirow{2}{*}{ Grupo estilístico } & \multicolumn{7}{|c|}{ Grupo petrográfico } & \multirow{2}{*}{ Total } \\
\cline { 2 - 8 } & A & B & F & D & C & E & P AT & \\
\hline I & $\mathbf{1 1}$ & $\mathbf{1 9}$ & 1 & & & & & 31 \\
II & 26 & 34 & & & & & & 60 \\
III & 1 & 1 & $\mathbf{2 1}$ & $\mathbf{1 6}$ & 2 & & 1 & $\mathbf{4 2}$ \\
VI & & & 3 & 9 & & & & 12 \\
V & 1 & & $\mathbf{1 1}$ & $\mathbf{1 3}$ & $\mathbf{4}$ & $\mathbf{7}$ & 1 & $\mathbf{3 7}$ \\
IV & & & & $\mathbf{1 1}$ & $\mathbf{1 4}$ & $\mathbf{1 6}$ & 2 & $\mathbf{4 3}$ \\
VII & 1 & 1 & 6 & $\mathbf{1 1}$ & $\mathbf{5 5}$ & $\mathbf{7 3}$ & $\mathbf{1 9}$ & $\mathbf{1 6 6}$ \\
E atípicos & 5 & 2 & 5 & & & & & 12 \\
\hline Total & 45 & 57 & 47 & 60 & 75 & 96 & 23 & 403 \\
\hline
\end{tabular}

\begin{tabular}{|c|c|c|c|c|c|c|c|c|}
\hline \multirow{2}{*}{ Grupo estilístico } & \multicolumn{7}{|c|}{ Grupo petrográfico } & \multirow{2}{*}{ Total } \\
\cline { 2 - 7 } & $\mathrm{A}$ & $\mathrm{B}$ & $\mathrm{F}$ & $\mathrm{D}$ & $\mathrm{C}$ & $\mathrm{E}$ & P AT & \\
\hline I & $\mathbf{3 5 \%}$ & $\mathbf{6 1 \%}$ & $3 \%$ & & & & & $100 \%$ \\
II & $43 \%$ & $57 \%$ & & & & & & $100 \%$ \\
III & $2 \%$ & $2 \%$ & $\mathbf{5 0} \%$ & $\mathbf{3 8} \%$ & $5 \%$ & & $2 \%$ & $\mathbf{1 0 0} \%$ \\
VI & & & $25 \%$ & $75 \%$ & & & & $100 \%$ \\
V & $3 \%$ & & $\mathbf{3 0} \%$ & $\mathbf{3 5} \%$ & $\mathbf{1 1} \%$ & $\mathbf{1 9} \%$ & $3 \%$ & $\mathbf{1 0 0} \%$ \\
IV & & & & $\mathbf{2 6} \%$ & $\mathbf{3 3} \%$ & $\mathbf{3 7 \%}$ & $5 \%$ & $\mathbf{1 0 0} \%$ \\
VII & $1 \%$ & $1 \%$ & $4 \%$ & $\mathbf{7 \%}$ & $\mathbf{3 3} \%$ & $\mathbf{4 4} \%$ & $\mathbf{1 1} \%$ & $\mathbf{1 0 0} \%$ \\
E AT & $42 \%$ & $17 \%$ & $42 \%$ & & & & & $100 \%$ \\
Total & $11 \%$ & $14 \%$ & $12 \%$ & $15 \%$ & $19 \%$ & $24 \%$ & $6 \%$ & $100 \%$ \\
\hline
\end{tabular}

\begin{tabular}{|c|c|c|c|c|c|c|c|c|}
\hline \multirow{2}{*}{ Grupo estilístico } & \multicolumn{7}{|c|}{ Grupo petrográfico } & \multirow{2}{*}{ Total } \\
\cline { 2 - 8 } & A & B & F & D & C & E & P AT & \\
\hline I & $\mathbf{2 4 \%}$ & $\mathbf{3 3} \%$ & $2 \%$ & & & & & $8 \%$ \\
II & $58 \%$ & $60 \%$ & & & & & & $15 \%$ \\
III & $2 \%$ & $2 \%$ & $\mathbf{4 5} \%$ & $\mathbf{2 7} \%$ & $3 \%$ & & $4 \%$ & $\mathbf{1 0 \%}$ \\
VI & & & $6 \%$ & $15 \%$ & & & & $3 \%$ \\
V & $2 \%$ & & $\mathbf{2 3} \%$ & $\mathbf{2 2} \%$ & $\mathbf{5 \%}$ & $\mathbf{7 \%}$ & $4 \%$ & $\mathbf{9 \%}$ \\
IV & & & & $\mathbf{1 8} \%$ & $\mathbf{1 9} \%$ & $\mathbf{1 7 \%}$ & $9 \%$ & $\mathbf{1 1 \%}$ \\
VII & $2 \%$ & $2 \%$ & $13 \%$ & $\mathbf{1 8} \%$ & $\mathbf{7 3} \%$ & $\mathbf{7 6} \%$ & $\mathbf{8 3} \%$ & $\mathbf{4 1 \%}$ \\
E AT & $11 \%$ & $4 \%$ & $11 \%$ & & & & & $3 \%$ \\
Total & $100 \%$ & $100 \%$ & $100 \%$ & $100 \%$ & $100 \%$ & $100 \%$ & $100 \%$ & $100 \%$ \\
\hline
\end{tabular}

Tabla 3. Frecuencias absolutas y relativas de los 403 fragmentos de vasijas de cerámica decoradas analizadas por grupos petrográficos y grupos estilisticos (J. Dulanto). 


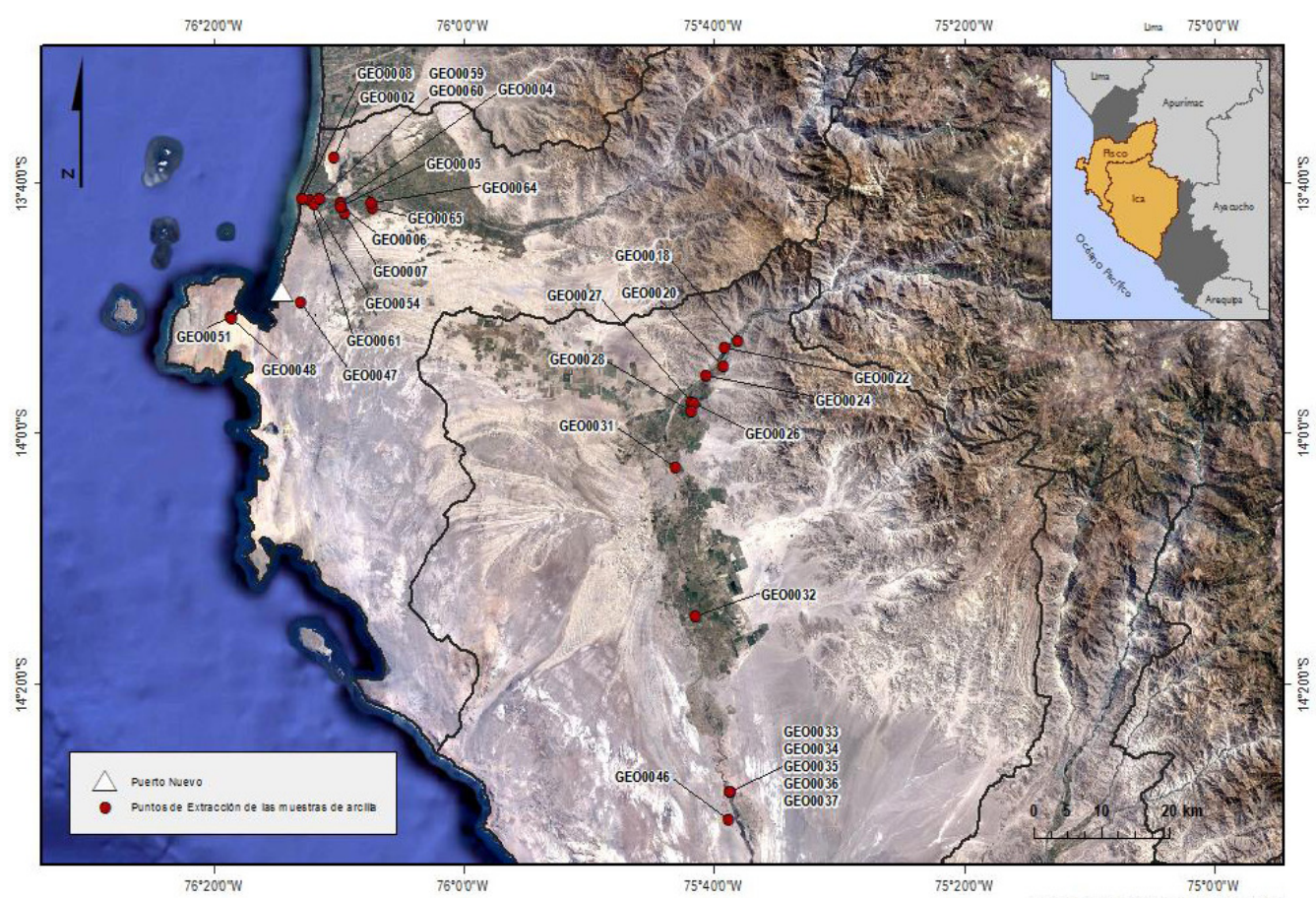

Figura 10. Ubicación de las muestras de arcilla recolectadas en los valles de los ríos Pisco e Ica (N. González).

6. Análisis de la composición química de los fragmentos de vasijas cerámica y muestras de arcilla por medio de LA-ICP-MS

Analizamos por medio de LA-ICP-MS la composición de 220 fragmentos de vasijas de cerámica y 19 muestras de arcilla del valle bajo de Pisco y el valle medio de Ica. Los análisis fueron llevados a cabo por Laure Dussubieux, Jalh Dulanto y Alejandro Rey de Castro en el Elemental Analysis Facility (EAF) del Field Museum of Natural History en Chicago, con un espectrómetro de masa Thermo ICAP Q acoplado a un láser New Wave UP213 para la ablación de las muestras.

El análisis por LA-ICP-MS permite identificar y cuantificar elementos mayores, menores y traza con altos niveles de detección, sin necesidad de preparar las muestras y sin destruirlas. Además, permite analizar la matriz de la arcilla, excluyendo las inclusiones ańadidas a esta. Para asegurar la representatividad de los datos, se mide la composición de la muestra en diez puntos distintos con un láser de 100 micrones ( 0.1 milímetros) de diámetro. Debido a que la matriz de la arcilla puede contener de manera natural limo y arena muy fina, el uso de una cámara CCD (charge coupled device image sensor) acoplada al láser permite elegir áreas que no contienen inclusiones gruesas. En la medida de lo posible las ablaciones son hechas sobre un corte fresco para evitar la contaminación de superficie. La composición de la muestra es el resultado del promedio de estas diez mediciones, después de eliminar aquellas anormales.

Se utilizó un estándar interno —el isótopo ${ }^{29} \mathrm{Si}$ - y las concentraciones para los elementos mayores, incluyendo el sílice, fueron calculadas asumiendo que el total de las concentraciones, en porcentaje del peso en vidrio, equivale a 100\% (Gratuze 1999). Dos estándares de materiales de referencia (NIST 679 y 610), con concentraciones conocidas, fueron utilizados para calcular las concentraciones de los elementos mayores y menores, y uno de ellos (NIST 610) fue empleado además para los elementos traza. Este último es un vidrio de silicato sodocálcico con elementos traza en concentraciones entre 400 y 500 ppm. Las concentraciones de Pearce et al. (1997) fueron 
usadas para los otros elementos. Para más detalles sobre la metodología ver Dussubieux et al. (2007), Golitko et al. (2012) y Sharratt et al. (2009).

El análisis de componentes principales (PCA) de la concentración de 50 elementos químicos en ppm de las 220 muestras de vasijas de cerámica y 19 muestras de fuentes de arcillas analizadas, permite explicar el $57.6 \%$ del total de la varianza, con los tres primeros componentes que, en ese orden, explican el 29.8\% (PC1), 18.0\% (PC2) y 9.76\% (PC3) de la varianza. Un examen de los diagramas de dispersión que comparan estos tres componentes (Figs. 11-13) permite identificar varios patrones generales y particulares.

Los patrones más claros que vale la pena resaltar incluyen: (1) la separación del conjunto de vasijas de los grupos petrográficos $\mathrm{A}$ y $\mathrm{B}$ de aquel de los grupos petrográficos $\mathrm{C}, \mathrm{D}, \mathrm{E}$ y $\mathrm{F}$ y los especímenes petrográficamente atípicos; y (2) la asociación del primero de estos conjuntos con las fuentes de arcilla del valle medio de Ica y el segundo de estos conjuntos con las fuentes de arcilla del valle bajo de Pisco.

En este punto es importante notar que la diferencia en la asociación entre estos dos conjuntos de vasijas de cerámica y las fuentes de arcilla podría ser el resultado de un sesgo en el muestreo de las fuentes de arcillas. Necesitamos incorporar al análisis muestras de fuentes del valle medio del río Pisco y del valle bajo del río Ica para esclarecer si esta diferencia en la asociación es entre valles (del río Pisco y del río Ica), entre secciones del valle (valle medio, valle bajo y litoral), o entre ambas. Cualquiera de estas dos posibilidades es interesante por las razones que exponemos en la siguiente sección.

Otros patrones que vale la pena resaltar incluyen: (3) una leve separación entre las vasijas del grupo petrográfico A y las del grupo petrográfico B; (4) una fuerte separación entre las vasijas de los grupos petrográficos A y B y las del grupo petrográfico C; (5) una leve sobreposición entre las vasijas de los grupos petrográficos A y $\mathrm{B}$ y las del grupo petrográfico $\mathrm{E}$; (6) una leve pero mayor sobreposición entre las vasijas de los grupos petrográficos A y $\mathrm{B}$ y aquellas de los grupos petrográficos D y F; y (7) una fuerte sobreposición entre las vasijas de los grupos petrográficos $\mathrm{C}$ y E, las de los grupos petrográficos D y F, y aquellas petrográficamente atípicas.

Es decir, los patrones de separación y sobreposición parcial de los grupos petrográficos en función de su composición química parecen replicar los patrones de separación y sobreposición parcial de estos mismos grupos petrográficos en función de sus características estilísticas. Como vimos en una sección anterior, las vasijas de los grupos petrográficos A y B tienden a ser vasijas de los grupos estilísticos I y II, las vasijas de los grupos petrográficos D y F tienden a ser vasijas de los grupos estilísticos III, V y VI, y las vasijas de los grupos petrográficos $\mathrm{C}$ y $\mathrm{E}$ tienden a ser vasijas de los grupos estilísticos IV y VII. Cierta sobreposición entre estos tres conjuntos se observa por la presencia de algunas vasijas del grupo petrográfico D que son vasijas de los grupos estilísticos IV y VII, y por la presencia de algunas vasijas de los grupos petrográficos $\mathrm{C}$ y $\mathrm{E}$ que son vasijas del grupo estilístico $\mathrm{V}$.

Finalmente, es interesante añadir que un análisis univariado simple de la concentración de ciertos elementos, nos permite reconocer algunos casos petrográficamente atípicos que son también químicamente atípicos. Un caso notable es el del espécimen PN2013-0232-0010, que muestra concentraciones inusualmente bajas de $\mathrm{Na}$, Ca y Sr, tal y como se muestra en la Figura 14. Este fragmento pertenece a una vasija que fue elaborada en un estilo característico de la costa extremo norte, ubicada a más de 1000 kilómetros al norte de Puerto Nuevo, y que es bien conocido a partir de materiales recolectados en prospecciones y excavaciones, especialmente en el valle de Piura (ver Bats 1990, 1991; Guffroy 1989, 1990, 1992, 1994; Hocquenhem y Kaulicke 1995; Kaulicke 1998).

Los otros fragmentos petrográficamente atípicos que, además, muestran concentraciones inusualmente altas o inusualmente bajas de alguno de los 50 elementos incluidos en el análisis de LAICPMS se presentan en la siguiente figura. Algunos de estos fragmentos revelan además peculiaridades estilísticas que los desvían de la norma dentro de los grupos estilísticos a los que fueron asignados. 


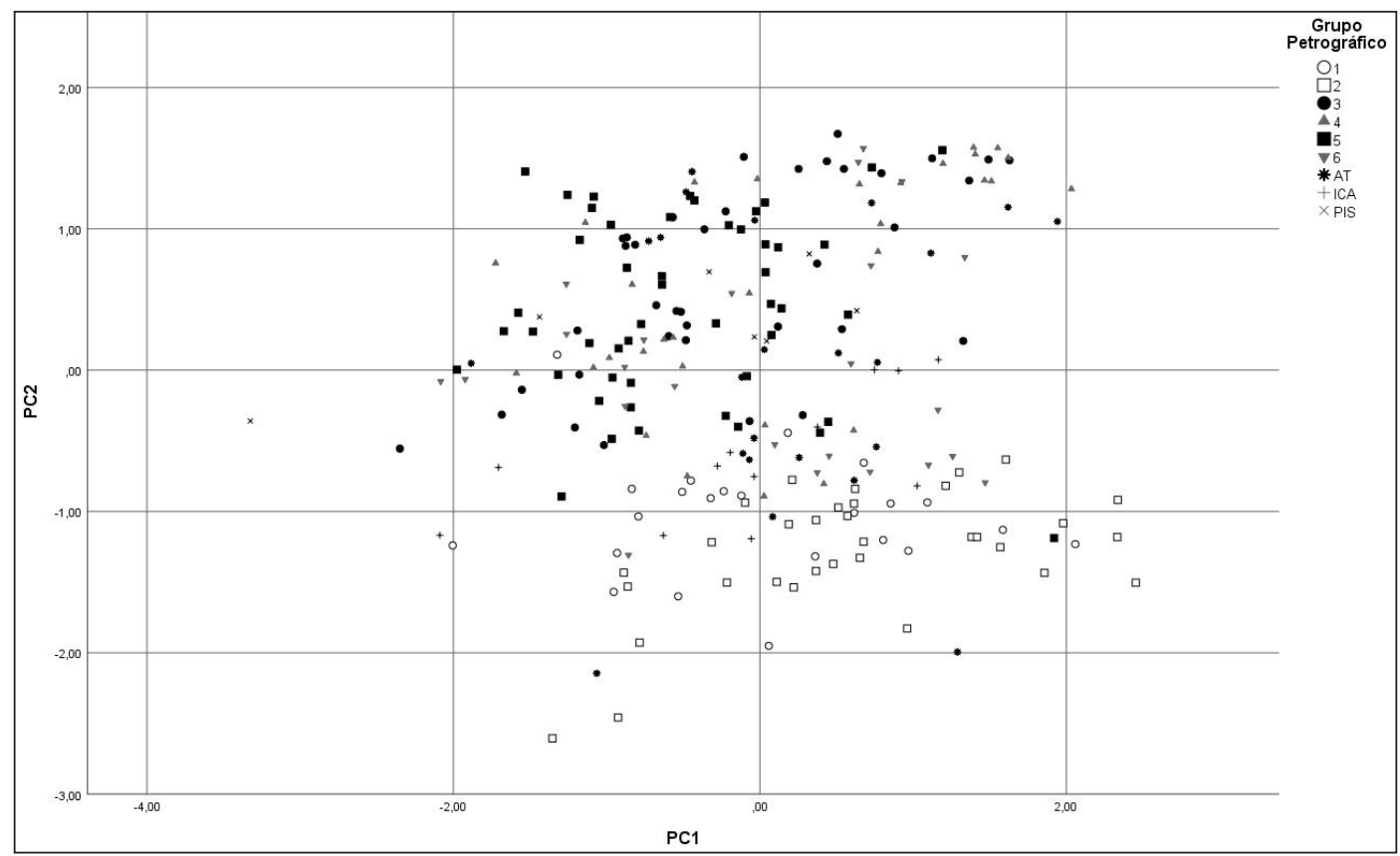

Figura 11. Resultados del análisis de componentes principales de la concentración de 50 elementos químicos para 220 fragmentos de vasijas de cerámica y 19 fuentes de arcilla de los ríos Pisco e Ica. PC1 vs. PC2 (J. Dulanto).

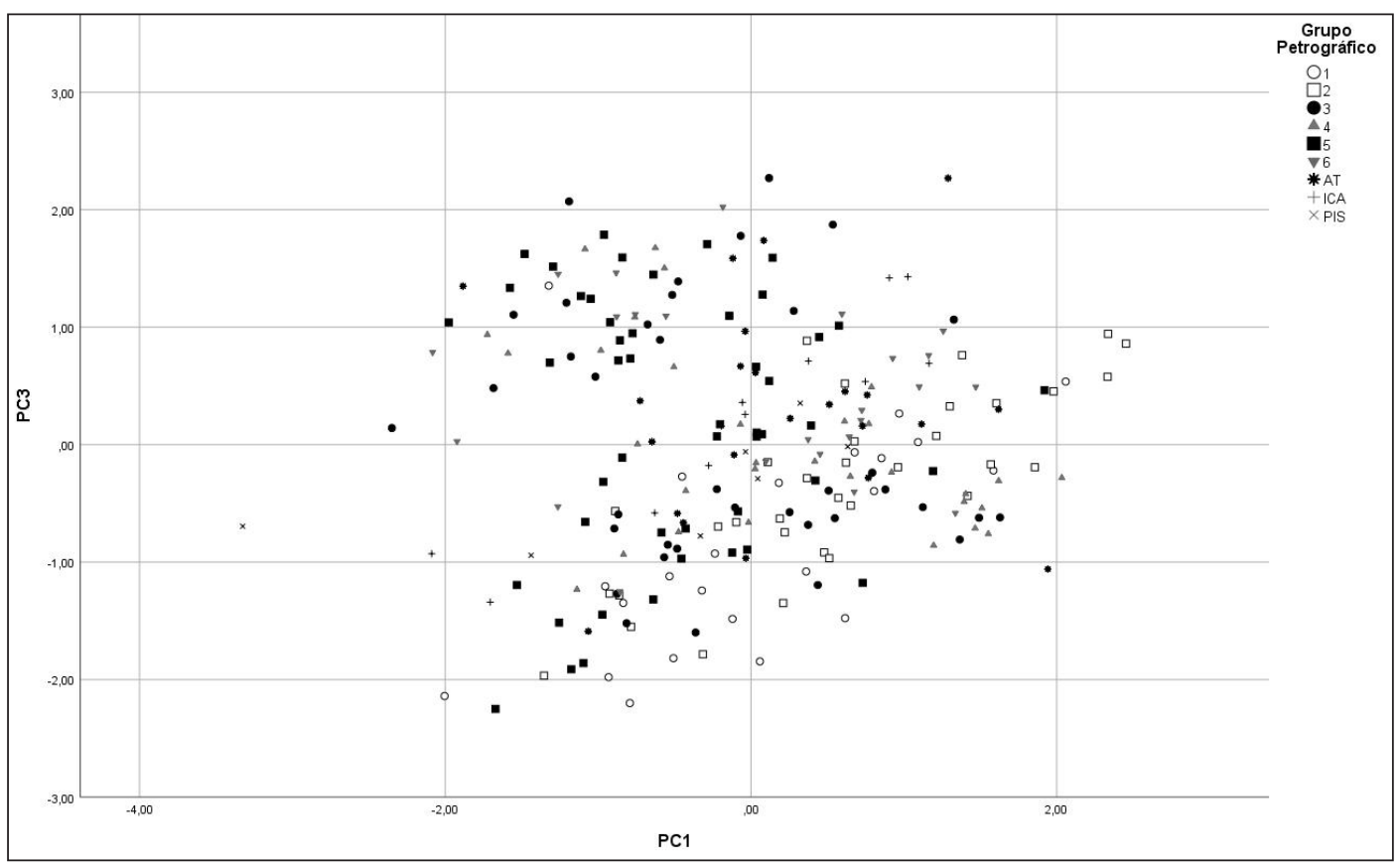

Figura 12. Resultados del análisis de componentes principales de la concentración de 50 elementos químicos para 220 fragmentos de vasijas de cerámica y 19 fuentes de arcilla de los ríos Pisco e Ica. PC1 vs. PC3 (J. Dulanto). 


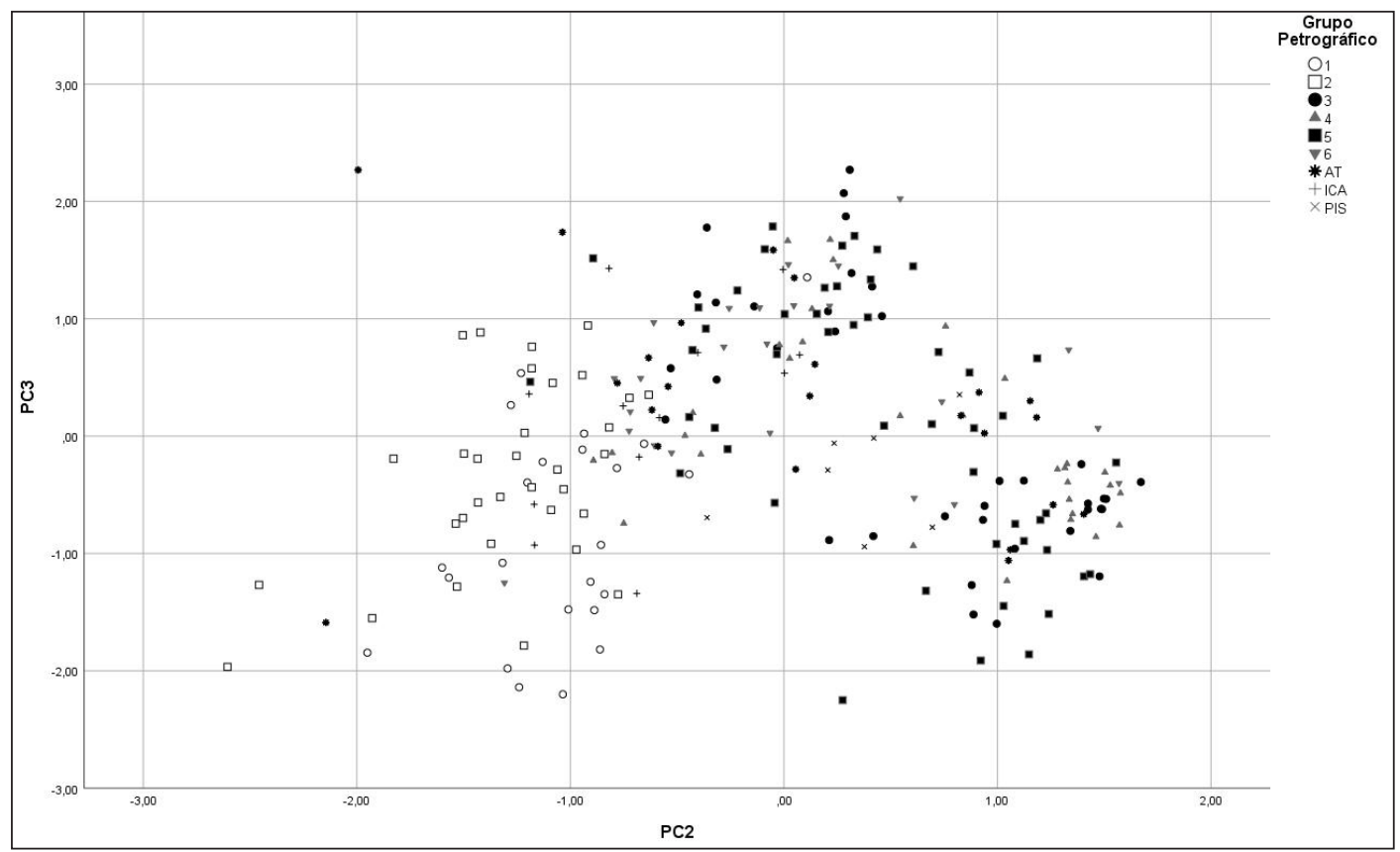

Figura 13. Resultados del análisis de componentes principales de la concentración de 50 elementos quimicos para 220 fragmentos de vasijas de cerámica y 19 fuentes de arcilla de los ríos Pisco e Ica. PC2 vs. PC3 (J. Dulanto).

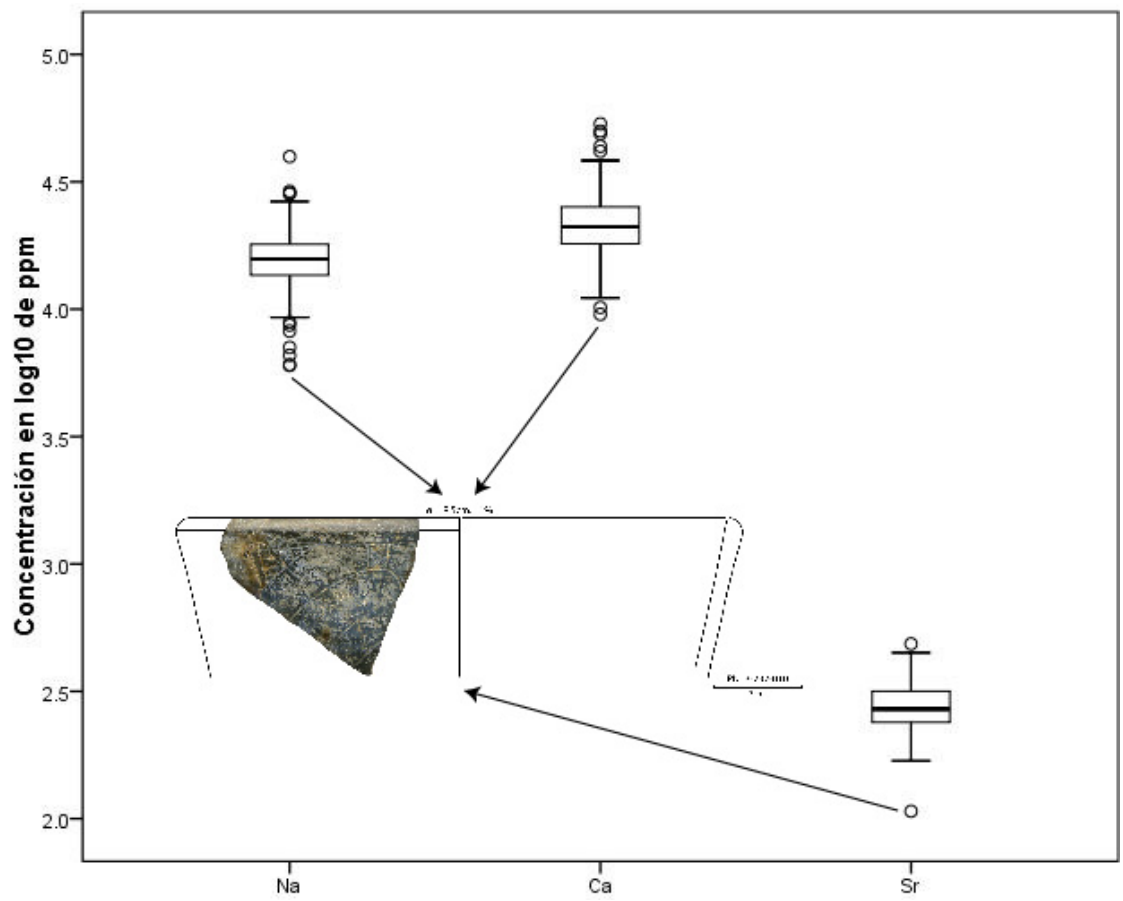

Figura 14. Diagramas de caja mostrando las concentraciones inusualmente bajas de Na, Ca y Sr del fragmento PN2013-0232-0010 (I. Aguirre). 
(1)
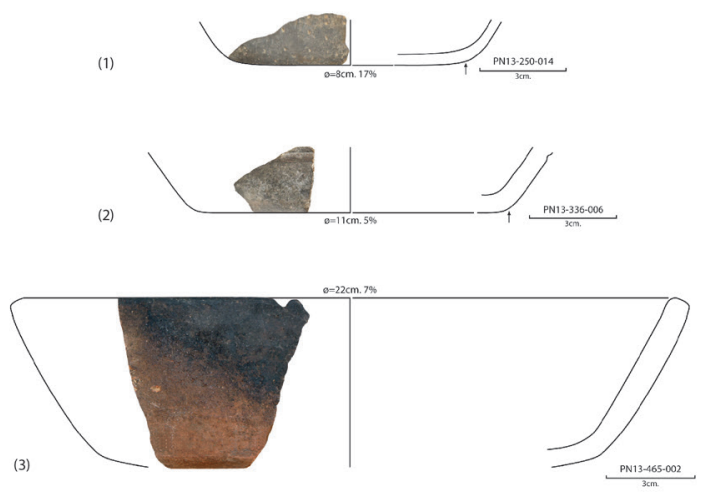

(4)
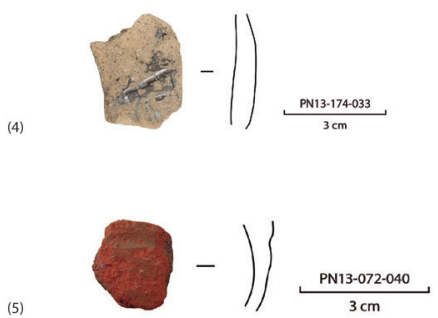
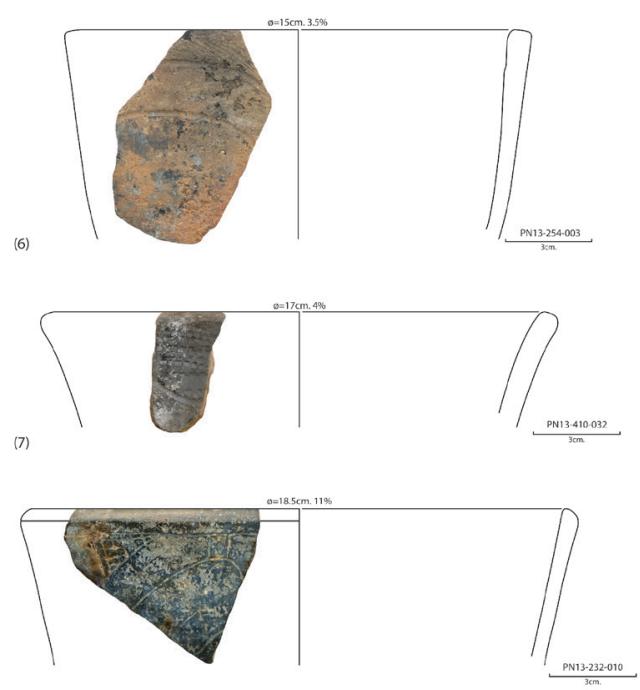

(9)

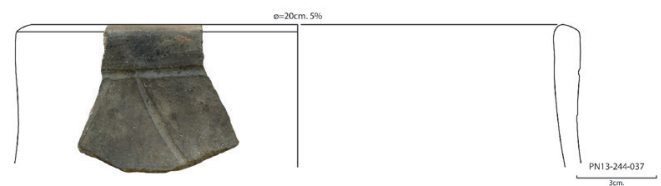

Figura 15. Fragmentos de vasijas petrográficamente atípicas que muestran concentraciones inusualmente altas o inusualmente bajas de algún elemento quimico (I. Aguirre).

\section{Conclusiones}

El análisis de la correlación entre la variación estilística y la variación composicional de las vasijas de cerámica de Puerto Nuevo permite responder varias preguntas que planteamos al inicio de este estudio, y también proponer nuevas interrogantes que pueden orientar esta investigación a futuro.

Tanto el análisis de la variación mineralógica como de la variación química en la composición de la arcilla con la que fueron manufacturadas estas vasijas sugiere fuertemente la existencia de tres fuentes de arcilla, o al menos tres áreas con estas fuentes. La primera de estas tres áreas está bien diferenciada químicamente de las otras dos, y está vinculada claramente a los grupos estilísticos I y II y a los grupos petrográficos A y B. Está ubicada muy probablemente en el valle medio o, en su defecto, en la sección más alta del valle bajo del río Ica, allí donde el valle se estrecha. Las otras dos áreas son más difíciles de diferenciar químicamente entre sí. La segunda de estas tres áreas está vinculada claramente a los grupos estilísticos III, VI y V y a los grupos petrográficos $\mathrm{F}$ y D, y está ubicada en el valle bajo del río Pisco, muy probablemente por encima de la tercera área. Finalmente, esta última está vinculada claramente a los grupos estilísticos V, IV y VII y a los grupos petrográficos $\mathrm{C}$ y E, y está ubicada también en el valle bajo del río Pisco, pero muy probablemente por debajo de la segunda área, muy cerca del litoral.

Es importante notar, sin embargo, que en este punto de la investigación es imposible determinar si la separación química de la primera área, por un lado, y la segunda y tercera áreas por el otro, refleja efectivamente una separación entre diferentes valles (valle del río Pisco y valle del río Ica), entre secciones del mismo valle (valle medio, valle bajo y litoral), o entre ambas. Para esto necesitamos incorporar en el análisis muestras de arcilla del valle medio del río Pisco y del valle bajo y litoral del río Ica. 
La primera de estas dos posibilidades es interesante porque los grupos estilísticos I y II muestran claros vínculos con estilos del valle de Ica hacia el sur, mientras que los grupos estilísticos III, V, VI y VII muestran vínculos evidentes con estilos del valle de Pisco hacia el norte. Teniendo en cuenta la ubicación de Puerto Nuevo en el área desértica de litoral, entre las desembocaduras de los ríos Pisco e Ica, podríamos estar frente a un caso de una zona de playa explotada simultáneamente - ya sea en forma permanente o estacionalmente_- por poblaciones vinculadas a estos dos valles.

La segunda de estas dos posibilidades, en cambio, es interesante porque los grupos IV y VII vinculados a las fuentes de arcilla ubicadas en las secciones más bajas del valle bajo adyacentes a la zona del litoral, son precisamente los que muestran claros vínculos con estilos costeños de zonas del litoral de diferentes regiones a lo largo de la costa al norte del valle de Pisco, mientras que los grupos estilísticos I y II vinculados a las fuentes de arcilla ubicadas en las secciones más altas del valle bajo, y las secciones más bajas del valle medio, son más bien los que muestran vínculos con estilos costeńos de zonas del interior de los valles al sur del valle de Pisco. Teniendo en cuenta la ubicación de Puerto Nuevo al borde del mar, podríamos estar frente a un caso de un asentamiento nodal, en el que confluyen redes de interacción que conectan a poblaciones distribuidas a lo largo del litoral y redes de interacción que conectan a poblaciones del litoral con las poblaciones del interior de los valles costeños e incluso con poblaciones de los valles serranos.

En ambos casos, las posibilidades de investigar la interacción de estas poblaciones a través de fronteras sociales, y la construcción de identidades producto de esa interacción, son múltiples especialmente teniendo en cuenta que los conjuntos de grupos estilísticos de vasijas de cerámica mencionados exhiben muchos préstamos tecnológicos, morfológicos y decorativos de estilos de otras regiones — hacia el Sur y hacia el Norte- - pero muy pocos préstamos entre sí. Para explorar estas interacciones, sin embargo, es necesario ampliar tanto la muestra de vasijas de cerámica, como la de fuentes de arcilla.

Por supuesto, cualquier exploración de la interacción entre diferentes poblaciones, ya sean de la misma región o de diferentes regiones, no puede perder de vista a los agentes involucrados en ella. En el caso de las vasijas de cerámica, el reto que enfrentamos los arqueólogos es sin dudas poder llegar a establecer si los productores, distribuidores y consumidores son los mismos agentes o son diferentes. Que la mayoría de las vasijas de cerámica de Puerto Nuevo que hemos estudiado hasta el momento hayan sido elaboradas con arcillas provenientes de fuentes locales y regionales (valles de Pisco e Ica), pero utilizando algunas materias primas foráneas (tal y como es el caso de algunos de los pigmentos utilizados para decorar vasijas de los grupos estilísticos IV y V, Dulanto et al. 2019), o copiando técnicas decorativas foráneas (tal y como es el caso de algunos tratamientos de superficie y técnicas de incisión y textura utilizados para decorar vasijas del grupo estilístico VII), o incluso adaptando motivos iconográficos foráneos (como es el caso de motivos empleados en la decoración de vasijas del grupo estilístico IV), sugiere que durante la primera mitad del primer milenio antes de nuestra era en la Costa Sur coexistieron y se sobrepusieron varios modos de interacción entre estas poblaciones. Algunos de estos lejos de ser excluyentes son complementarios, y podrían incluir no solo la importación de vasijas de otras regiones y la copia de estas vasijas importadas por alfareros locales, sino también — tal y como ha sido mostrado sugerentemente para los Andes Centrales por Gabriel Ramón (2013) — la presencia de alfareros itinerantes de otras regiones, que producen vasijas en estilos foráneos y locales. Este último modo cobra particular fuerza cuando observamos el grado de similitud entre varias de las vasijas del grupo estilístico VII, y aquellas que conocemos por el trabajo de otros investigadores para sitios del litoral de la Costa Central como Ancón y Curayacu. 


\section{Notas}

${ }^{1}$ Definimos la costa surcentral del Perú como el área comprendida por los valles de Chincha, Pisco, Ica y Nasca del departamento de Ica, y las regiones naturales de Chala (0-500 msnm) y Yunga Marítima (500-2300 msnm) según Pulgar Vidal (1987).

${ }^{2}$ En publicaciones anteriores (Druc et al. 2017) hemos considerado menos o más fragmentos de vasijas de cerámica como decorados en función de considerar o no como decoración ciertas formas de los cuerpos o ciertos tratamientos de superficie de las vasijas. Sin embargo, estos cambios no alteran los patrones observados, ni en los resultados del análisis estilístico, ni en los del análisis petrográfico.

\section{REFERENCIAS}

Bats, J. C. (1990). La prospection systématique de la basse vallée du Yapatera: approche typologique et classification du matériel céramique, DEA, Université de Paris 1.

Bats, J. C. (1991). Ruptures et continuités culturelles dans la basse vallée du Yapatera: approche typologique formalisée d 'un maté riel céramique recolté en prospection, Bulletin de l' Institut Francais d 'Etudes Andines 20 (2), 349-380.

Druc, I., J. Dulanto, E. Guadalupe y A. Rey de Castro (2017). Análisis de la composición mineral de las vasijas de cerámica de Puerto Nuevo: algunas consideraciones preliminares sobre su producción y procedencia, Boletín de Arqueología PUCP 22, 133-157.

Dulanto, J. (2013). Puerto Nuevo: Redes de intercambio a larga distancia durante la primera mitad del primer milenio antes de nuestra era, Boletín de Arqueología PUCP 17, 103-132.

Dulanto, J. (e.p.). The Chavín sphere of interaction and Peru's south coast: maritime communities, long distance exchange networks, and prestige economies during the Early Horizon in the central Andes, en: R. Burger y J. Nesbitt (eds.), Reconsidering the Chavin Phenomenon in the 21st Century, 1-25, Dumbarton Oaks Research Library and Collection, Washington, D.C.

Dulanto, J. y A. Accinelli (2013). Disco Verde 50 años después de Frédéric Engel: La primera temporada de excavaciones del Proyecto de Investigaciones Arqueológicas Paracas en el sitio, Boletín de Arqueología PUCP 17, 133-150.

Dulanto, J., P. Gonzales y E. Guadalupe (2019). Los pigmentos utilizados en la pintura postcocción de vasijas de cerámica Paracas Temprano de Puerto Nuevo y las redes de intercambio de la costa y sierra surcentral del Perú durante los siglos IX-VI a.C., Boletín de Arqueología PUCP 26, 65-83.

Dussubieux, L., M. Golitko, P. Ryan Williams y R. J. Speakman (2007). Laser ablation-inductively coupled plasma-mass spectrometry analysis applied to the characterization of Peruvian Wari ceramics, en: M. D. Glascock, R.J. Speakman y R. S. Popelka-Filcoff (eds.), Archaeological chemistry: Analytical techniques and archaeological interpretation, 349-363, American Chemical Society, Washington, D.C.

Engel, F. (1963a). Datations à l'aide du radio-carbone 14 et problèmes de la préhistoire du Pérou, Journal de la Société des Américanistes 52, 101-132 https://doi.org/10.3406/jsa.1963.1995

Engel, F. (1963b). Notes relatives à des explorations archéologiques à Paracas et sur la côte sud du Pérou, Travaux de l'Institut Français d'Études Andines 9, 1-72.

Engel, F. (1963c). A preceramic settlement on the central coast of Peru: Asia, unit 1, Transactions of the American Philosophical Society 53(3), 1-139. https://doi.org/10.2307/1005956

Engel, F. (1966). Paracas: cien siglos de cultura peruana. Editorial Juan Mejía Baca, Lima.

Engel, F. (1976). An ancient world preserved: Relics and records of prehistory in the Andes, Crown Publishers, Nueva York.

Engel, F. (1987). De las begonias al maiz: Vida y Producción en el Perú Antiguo, Centro de Investigación de Zonas Áridas, Universidad Nacional Agraria, Lima.

Engel, F. (1991). Un desierto en tiempos prehispánicos, Foundation pour L'Etude des Problemes de Terres Arides, Lima.

García, R. (2009). Puerto Nuevo y los orígenes de la tradición estilístico-religiosa Paracas, Boletín de arqueología PUCP 13, 187-207.

García, R. y J. Pinilla (1995). Aproximación a una secuencia de fases con cerámica temprana de la región de Paracas, Journal of the Steward Anthropological Society 23, 43-81. 
Golitko, M., J. Dudgeon, H. Neff y J. E. Terrell (2012). Identification of post-depositional chemical alteration of ceramics from the North Coast of New Guinea (Sandaun Province) by Time of Flight-Laser-AblationInductively Coupled Plasma-Mass Spectrometry (TOF-LA-ICP-MS), Archaeometry 54(1), 80-100. https:// doi.org/10.1111/j.1475-4754.2011.00612.x

Gratuze, B. (1999). Obsidian characterization by laser ablation ICP-MS and its application to prehistoric trade in the Mediterranean and the Near East: Sources and distribution of obsidian within the Aegean and Anatolia, Journal of Archaeological Science 26, 869-881. https://doi.org/10.1006/jasc.1999.0459

Guffroy, J. (1989). Un centro ceremonial formativo en el Alto Piura, Bulletin de l'Institut Francais d'Etudes Andines 18(2), 161-207.

Guffroy, J. (1990). Le développement des premieres grandes civilizations andines dans 1 'extreme nord du Pérou, Cabiers des Sciences Humaines 26(4), 623-654.

Guffroy, J. (1992). Las tradiciones culturales formativas en el Alto Piura, en: D. Bonavia (ed.), Estudios de Arqueología Peruana, 99-122, FOMCIENCIAS, Lima.

Guffroy, J. (1994). Cerro Nañañique: Un établissement monumental de la période formative, en limite de désert (Haut Piura,Pérou), ORSTOM, París.

Hocquenhem, A. M. y P. Kaulicke (1995). Estudio de una colección de cerámica de Yacila, extremo norte del Perú, Bulletin de l'nstitut Francais d'Etudes Andines 24(2), 213-243.

Kaulicke, P. (1998). El formativo de Piura, Boletín de Arqueologia PUCP 2, 19-36.

Pearce, N. J. G., W.T. Perkins, J.A. Westgate, M.T. Gorton, S.E. Jackson, C.R. Neal y S. P. Chenery (1997).A compilation of new and published major and trace element data for NIST SRM 610 and SRM 612 glass reference materials, Geostandards Newsletter 22, 114-115.

Pulgar Vidal, J. (1987). Geografia del Perú: Las Ocho Regiones Naturales del Perú, Peisa, Lima.

Ramón, G. (2013). Los Alfareros Golondrinos: Productores Itinerantes en los Andes, IFEA y Sequilao Editores, Lima.

Sharratt, N., M. Golitko, P. Ryan Williams y L. Dussubieux (2009). Ceramic production during the Middle Horizon: Wari and Tiwanaku clay procurement in the Moquegua Valley, Peru, Geoarchaeology 24(6), 792-820. https://doi.org/10.1002/gea.20288

Fecha de recepción: 5-8-2019

Fecha de aceptación: 16-12-2019 